\title{
Musicoterapia Improvisacional
} Musicocentrada e Crianças com Autismo: Relações entre Desenvolvimento Musical, Ganhos Terapêuticos e a Teoria da Musicalidade Comunicativa

\author{
Music-centered Improvisational Music Therapy \\ and Children with Autism: Relations between \\ Musical Development, Therapeutic Gains, and the \\ Communicative Musicality Theory
}

\footnotetext{
(D) Marina Horta Freire

Universidade Federal de Minas Gerais, Programa de Pós-graduação em Música, Belo Horizonte, MG, Brasil

marinahf@gmail.com

iD Maria Betânia Parizzi

Universidade Federal de Minas Gerais, Programa de Pós-graduação em Música, Belo Horizonte, MG, Brasil

betaniaparizzi@hotmail.com

iD Jéssica Martelli

Universidade Federal de Minas Gerais, Licenciatura em Música, Belo Horizonte, MG, Brasil jessicamartelli7@gmail.com

iD Renato Tocantins Sampaio

Universidade Federal de Minas Gerais, Programa de Pós-graduação em Música, Belo Horizonte, MG, Brasil

renatots@musica.ufmg.br
}

\begin{abstract}
Resumo: O presente artigo aborda as interfaces entre Musicoterapia, desenvolvimento musical e Musicalidade Comunicativa, com o objetivo de investigar o desenvolvimento musical de crianças com autismo e suas relações com ganhos terapêuticos em Musicoterapia Improvisacional
\end{abstract}


Musicocentrada. Para isto, foi avaliado o desenvolvimento musical de 25 crianças com autismo por meio da Escala DEMUCA (FREIRE et al., 2019), no início e no final do tratamento de Musicoterapia, e verificadas possíveis correlações com ganhos terapêuticos mensurados por meio das escalas ATEC, CGAS e CGI (FREIRE, 2014). Os resultados mostraram maior nível de desenvolvimento musical após o tratamento e correlações positivas do desenvolvimento musical com melhoras, principalmente na linguagem/comunicação. Dentre as discussões levantadas, destacou-se a relevância da teoria da Musicalidade Comunicativa para se compreender as trocas musicais afetivas entre terapeuta e paciente que podem levar à expansão da comunicação e do desenvolvimento musical do paciente. Conclui-se que, por essa proximidade da Musicoterapia com a Musicalidade Comunicativa, é possível apontar conexões entre ganhos terapêuticos e desenvolvimento musical de crianças com autismo atendidas em Musicoterapia Improvisacional Musicocentrada.

Palavras-chave: Musicoterapia. Desenvolvimento Musical. Musicalidade Comunicativa. Autismo.

\begin{abstract}
Thisarticleaddresses interfaces between MusicTherapy, musical development, and Communicative Musicality, aiming to investigate the musical development of children with autism and their relationship with therapeutic gains in Music-centered Improvisational Music Therapy. For that, the musical development of 25 children with autism at the beginning and end of the Music Therapy treatment was evaluated through DEMUCA Scale (FREIRE et al., 2019), and was correlated with the therapeutic gains mensured through the scales ATEC, CGAS, and CGI (FREIRE, 2014). The results showed a higher level of musical development after treatment and positive correlations of musical development with improvements mainly in language/communication. Among the discussions raised, the relevance of the theory of Communicative Musicality was highlighted in order to understand the affective musical exchanges between therapist and patient that can lead to the expansion of communication and the musical development of the patient. We conclude that, due to the proximity of Music Therapy to Communicative Musicality, it is possible to point
\end{abstract}


out connections between therapeutic gains and musical development of children with autism treated in Music-centered Improvisational Music Therapy.

Keywords: Music Therapy. Musical development. Communicative Musicality. Autism

Submetido em: 27 de fevereiro de 2020

Aceito em: 17 de março de 2021 
Musicoterapia Improvisacional Musicocentrada e Crianças com Autismo: Relações... Marina Horta Freire • Maria Betânia Parizzi • Jéssica Martelli • Renato Tocantins Sampaio

\section{Introdução}

Esta seção introdutória traz o conceito de Desenvolvimento Musical, apresentando a Teoria da Musicalidade Comunicativa com a finalidade de conduzir o leitor à compreensão das relações desses conceitos com o Autismo e a Musicoterapia, e de fundamentar as análises e discussões apresentadas neste presente estudo.

O Desenvolvimento Musical pode ser entendido como um processo de transformação no fazer musical de um indivíduo, ao longo do tempo, pelo aprimoramento de habilidades em atividades específicas da área da música, que pode ser alcançado por meio da educação musical formal ou do contato natural e espontâneo com a música (COVRE, 2015). Compreendido também como um importante indicador do desenvolvimento do indivíduo, o desenvolvimento musical é caracterizado como processo progressivo que agrupa habilidades sensório-motoras, cognitivas e de interação social. Por sua interdependência com diversos fatores culturais, sociais e biológicos, o desenvolvimento musical não se apresenta em etapas ou estágios fixos e pré-definidos (NORTH; HARGREAVES, 2008; PENDEZA; AZEVEDO, 2018).

É importante colocar que a prática musical, fundamental para o desenvolvimento musical do indivíduo, desempenha também papel relevante no desenvolvimento das funções neurológicas em geral, pois estimula simultaneamente diferentes regiões cerebrais (ROCHA; BOGGIO, 2013; CUERVO; ROSAT, 2018). As habilidades cognitivas (percepção sensorial, atenção, memória, criatividade etc.) e sociais são particularmente estimuladas ao longo da aprendizagem musical, contribuindo assim para o desenvolvimento integral da pessoa (MORENO et al., 2011; SANTOS, 2014).

A Teoria da Musicalidade Comunicativa (MALLOCH, 1999/2000) teve sua origem em pesquisas fundamentadas na observação do comportamento de mães e de seus bebês durante momentos de interação da díade. Neste engajamento afetivo, mãe e bebê estabelecem uma "protoconversação", que apresenta características 
Musicoterapia Improvisacional Musicocentrada e Crianças com Autismo: Relações... Marina Horta Freire • Maria Betânia Parizzi • Jéssica Martelli • Renato Tocantins Sampaio

presentes na música como: pulso (sucessão regular de etapas comportamentais distintas, expressas nas trocas de turno características da interação mãe/bebê), "qualidades expressivas" (movimentos melódicos, variações timbrísticas e gestualidade expressiva da díade utilizados nos momentos de interação) e narrativas (sentido construído a partir do pulso e das "qualidades expressivas", que permite que adulto e bebê, ou adulto e adulto, compartilhem suas subjetividades em um mesmo lapso de tempo) (MALLOCH; TREVARTHEN, 2009).

Essas características da música, presentes na voz, na mímica facial e nos movimentos corporais têm, por si só, uma função comunicativa que persiste nas interações sociais posteriores. Por isso, sendo a primeira e mais primordial forma de interação/comunicação humana, a Musicalidade Comunicativa pode ser considerada um importante fator responsável pela sobrevivência e desenvolvimento do indivíduo, que fundamentará a autoconsciência, a autorregulação, a intersubjetividade e o engajamento solidário ao longo da vida (MALLOCH; TREVARTHEN, 2009; MALLOCH; TREVARTHEN, 2018).

\begin{abstract}
A musicalidade da interação vocal entre mãe e bebê é vantajosa para a sobrevivência do bebê e para o vínculo da díade, contribuindo a manter e regular a atenção do bebê, favorecendo o desenvolvimento da estrutura linguística, e promovendo a comunicação das emoções e a regulação do comportamento social (PALAZZI, 2016, p. 19).
\end{abstract}

De acordo com Covre (2015), a Musicalidade Comunicativa age como um sinal social do aprendizado da criança, sendo essencial na regulação emocional, na formação de vínculos, na orientação da atenção e na facilitação da percepção dos sons e da linguagem. As relações pré-musicais, estabelecidas por mãe-bebê, auxiliam a criança a futuramente apreender a língua e a música de sua cultura (ANSDELL, 2014). Além disso, a própria música, por compartilhar com a Musicalidade Comunicativa algumas de suas características, 
Musicoterapia Improvisacional Musicocentrada e Crianças com Autismo: Relações... Marina Horta Freire • Maria Betânia Parizzi • Jéssica Martelli • Renato Tocantins Sampaio

é capaz de proporcionar situações comunicativas que podem favorecer o desenvolvimento tanto da linguagem como de habilidades musicais (COVRE, 2015).

\section{Tecendo relações entre Autismo, Musicoterapia, Desenvolvimento Musical e Musicalidade Comunicativa}

A condição clínica do autismo (ou Transtorno do Espectro do Autismo, em acordo com o DSM-5 - APA, 2014) é caracterizada por dificuldades sociocomunicacionais e por padrões comportamentais restritos e repetitivos, que se apresentam em diferentes níveis de gravidade de indivíduo para indivíduo. Além dessas características, pessoas com autismo destacam-se por estabelecerem relações peculiares com a música, considerada como uma janela única para o mundo do autismo (MOLNAR-SZAKACS; HEATON, 2012; MALLOCH e TREVARTHEN, 2018). Em estudos sobre o autismo, a música é reconhecida como provocadora de respostas e interesses não usuais (THAUT, 1988; THAUT, 2008), um importante recurso motivacional (TREVARTHEN, 2002; MALLOCH e TREVARTHEN, 2018) e eficaz ferramenta terapêutica (GERETSEGGER et al., 2014; MÖSSLER et al., 2019).

Em relação às habilidades musicais, indivíduos com autismo podem demonstrar uma elevada sensibilidade na percepção de alturas e timbres, e ter facilidade em reconhecer estruturas musicais e o caráter expressivo de obras musicais (FIGUEIREDO, 2016), podendo apresentar ouvido absoluto (OUIMET et al., 2012) e habilidades motoras acima da média para tocar instrumentos musicais (MOLNAR-SZAKACS; HEATON, 2012).

Por todos esses motivos, o desenvolvimento musical pode influenciar positivamente o desenvolvimento das funções neurológicas da pessoa com autismo. Esse processo parece estar relacionado ao incremento da neuroplasticidade (principalmente nas áreas cerebrais associadas à motricidade, linguagem e emoções) (WAN; 
Musicoterapia Improvisacional Musicocentrada e Crianças com Autismo: Relações... Marina Horta Freire • Maria Betânia Parizzi • Jéssica Martelli • Renato Tocantins Sampaio

SCHALAUG, 2010) e de neurônios-espelho. Essas modificações neurológicas parecem contribuir para a melhora da autonomia e da capacidade de tomada de decisões (LOURO; 2012), aprimorando o convívio social do autista (MOLNAR-SZAKCS et al., 2009).

No campo da Musicoterapia também são mostradas importantes evoluções de crianças com autismo, principalmente em tratamentos musicoterapêuticos individuais, destacando-se meIhoras nas habilidades comunicacionais, nas interações sociais e redução de comportamentos estereotipados (FINNIGAN; STARR, 2010; SIMPSON; KEEN, 2011; SAMPAIO, 2015; ROJAS et al., 2018). Em Musicoterapia Improvisacional, que é a abordagem mais comumente encontrada nas pesquisas e na prática clínica em autismo (GATTINO, 2012), estudos mostram ganhos terapêuticos relevantes das crianças atendidas, tais como: melhora na atenção conjunta e na imitação (KIM et al., 2008), diminuição de comportamentos indesejáveis como choro e estereotipias vocais (KIM et al., 2009) e melhora na comunicação não verbal (GATTINO, 2012; SARAPA; KATUSIC, 2012).

Podem ser estabelecidas importantes conexões entre esses ganhos terapêuticos de crianças com autismo e a teoria da Musicalidade Comunicativa (MALLOCH; TREVARTHEN, 2018). As técnicas de improvisação musical clínica permitem ao musicoterapeuta usar os parâmetros da Musicalidade Comunicativa (MALLOCH; TREVARTHEN, 2018), de forma sensível e consciente, para mobilizar e apoiar as peculiaridades sonoras utilizadas pelo paciente para se expressar, a fim de estabelecer conexão, comunicação e interação (TREVARTHEN, 2002; ANSDELL, 2014; MALLOCH; TREVARTHEN, 2018). Essa mobilização e esse apoio são essenciais, pois crianças com autismo podem apresentar, desde o nascimento, dificuldades em compreender e em se expressar durante suas interações, o que dificulta o engajamento intersubjetivo e pode resultar no desenvolvimento atípico da comunicação social (TREVARTHEN, 2002; WIGRAM; ELEFANT, 2009; MALLOCH; TREVARTHEN, 2018;). 
Musicoterapia Improvisacional Musicocentrada e Crianças com Autismo: Relações... Marina Horta Freire • Maria Betânia Parizzi • Jéssica Martelli • Renato Tocantins Sampaio

Os musicoterapeutas Robarts (1998) e Wigram e Elefant (2009) relatam casos de crianças com autismo que conseguiram, por meio da improvisação musical clínica coativa', descobrir, explorar e desenvolver seu potencial de comunicação, melhorando sua comunicação empática, interação social, confiança e afetividade com outras pessoas fora da terapia. Assim, indo ao encontro da musicalidade do paciente, o processo musicoterapêutico improvisacional facilita o engajamento sonoro-comunicativo, espontâneo e afetivo, o que permite à criança com autismo vivenciar experiências criativas e desenvolver intersubjetividade, autorregulação, auto-organização e consciência de si e do outro (ROBARTS, 1998; TREVARTHEN, 2002; WIGRAM; ELEFANT, 2009; ANSDELL, 2014; MALLOCH; TREVARTHEN, 2018).

Alguns autores da Musicoterapia apontam que, no processo musicoterapêutico, as melhoras clínicas também podem ser observadas na evolução da produção musical do paciente, que passa a manifestar comportamentos musicais aprimorados e inéditos (BARCELLOS, 2009; SANTOS, 2018). Sampaio (2015) mostra que conquistas manifestadas no fazer musical entre musicoterapeuta e paciente, como a melhora da sincronicidade rítmica, é uma forma de se observar o desenvolvimento comunicacional de pessoas com autismo. De fato, parâmetros do desenvolvimento musical, como, por exemplo, regulação temporal ${ }^{2}$ e senso de conclusão ${ }^{3}$, podem ser considerados referências para avaliação e acompanhamento do processo musicoterapêutico (NORDOFF; ROBBINS, 2007; SAMPAIO, 2015; PANDEZA; AZEVEDO, 2018).

Todavia, pesquisas experimentais que enfatizam a importância do desenvolvimento musical em sessões musicoterapêuticas de pessoas com autismo ainda são escassas na literatura científica, especialmente no Brasil (SAMPAIO, 2015; FREIRE et al., 2018). Santos (2018) avaliou qualitativamente o desenvolvimento de habilidades

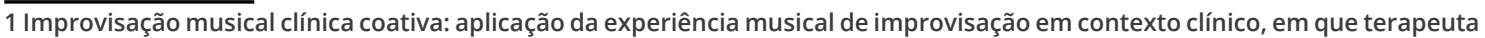
e paciente improvisam juntos, sendo o foco central da Musicoterapia Improvisacional (FREIRE, 2019).

2 Regulação temporal: consiste no ajuste motor à pulsação proveniente do ambiente externo. A criança adapta seu gesto à pulsação da música, ou seja, regula suas "batidas" à pulsação da música que ouve. (CARNEIRO; PARIZZI, 2011).

3 Senso de conclusão: a criança demonstra ter a noção de direção do discurso musical, finalizando frases musicais com recursos rítmico-sonoros diversos (PARIZZI, 2009).
} 
Musicoterapia Improvisacional Musicocentrada e Crianças com Autismo: Relações... Marina Horta Freire • Maria Betânia Parizzi • Jéssica Martelli • Renato Tocantins Sampaio

musicais de crianças com autismo em sessões musicais integradas de Musicoterapia Interativa e Educação Musical, relacionando os processos musicais com processos psicológicos, que auxiliam no desenvolvimento integral da criança com autismo. Já em um estudo de caso único, autores do presente artigo mostraram o desenvolvimento musical de um menino com autismo atendido em Musicoterapia. Apontaram que os seus ganhos terapêuticos já comprovados em pesquisa anterior (FREIRE, 2014), principalmente a melhora na comunicação e interação social, poderiam estar relacionados ao desenvolvimento musical alcançado pela criança no decorrer do processo musicoterapêutico (FREIRE et al., 2018). Esse estudo de caso utilizou a Escala de Desenvolvimento Musical de Crianças com Autismo (FREIRE et al., 2019) na mensuração do desenvolvimento musical do paciente.

A partir desse contexto, a proposta dos autores deste presente artigo foi dar continuidade às pesquisas anteriores (FREIRE, 2014; FREIRE et al., 2018; FREIRE et al., 2019), tendo como objetivos: (1) investigar o desenvolvimento musical de um grupo de crianças com autismo, atendidas em Musicoterapia Improvisacional Musicocentrada, e (2) pesquisar as possíveis relações entre o desenvolvimento musical encontrado e os ganhos terapêuticos alcançados com essas mesmas crianças.

Para isso, a presente pesquisa utiliza vídeos (dados pré-coletados) de 25 crianças com autismo, atendidas previamente, durante a realização do Mestrado “Efeitos da Musicoterapia Improvisacional no Tratamento de Crianças com Transtorno do Espectro do Autismo", defendido no Programa de Pós-Graduação em Neurociências da UFMG (FREIRE, 2014). A fim de compreender esse contexto, apresentamos a seguir um resumo da pesquisa prévia, antes de descrever a metodologia da presente pesquisa. 


\section{PesquisaPrévia-Participantes, IntervençãoeResultados ${ }^{4}$}

Vinte e cinco crianças com autismo foram atendidas em Musicoterapia Improvisacional Musicocentrada, entre 2012 e 2014, no Ambulatório do Serviço de Psiquiatria do Hospital das Clínicas da UFMG. Dentre essas crianças, 22 eram meninos e 3 eram meninas, que tinham entre 3 e 6 anos de idade durante os atendimentos.

Cada criança teve 15 sessões individuais e semanais de Musicoterapia Improvisacional Musicocentrada, com 30 minutos de duração cada. Nesta abordagem musicoterapêutica, priorizou-se o fazer musical conjunto, a partir das iniciativas sonoras e/ou musicais do próprio paciente, seguidas de espelhamento, sustentação e encorajamento musicais do terapeuta (FREIRE et al., 2015). O musicoterapeuta buscava criar um espaço lúdico de exploração de instrumentos e da voz, com foco principal na expressividade musical e no fortalecimento do vínculo musical-terapêutico, com o objetivo de desenvolver potenciais latentes da criança, facilitando a melhora da comunicação e da interação (FREIRE et al., 2015). Assim, a abordagem musicoterapêutica realçava a importância da música e da relação musical estabelecida entre terapeuta e paciente. Porém, as intervenções não envolviam objetivos focados no desenvolvimento musical das crianças atendidas, e sim no desenvolvimento de sua comunicação social.

As crianças passaram por avaliação inicial e final (antes e após o processo musicoterapêutico), por meio de entrevista e questionários respondidos por seus pais/responsáveis, uma semana antes da primeira sessão e uma semana após a última sessão de cada criança. As avaliações foram conduzidas pela própria musicoterapeuta e tiveram duração de uma hora cada. Nessas avaliações, foram coletados dados referentes à comunicação, socialização, cognição, comportamentos estereotipados e grau de funcionalidade da criança em escalas próprias para este fim (que serão explicadas mais adiante). Outras 20 crianças passaram pelas mesmas

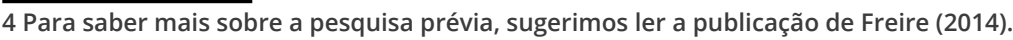


avaliações, mas não receberam as sessões de Musicoterapia, participando de grupo controle.

Os dados coletados nas avaliações iniciais foram comparados quantitativamente com os coletados nas avaliações finais por meio de análises estatísticas. Os resultados mostraram melhoras significativas do grupo de crianças atendidas, comparadas a grupo controle, em todas as áreas do desenvolvimento afetadas pelo autismo: comunicação, socialização, funcionalidade e comportamentos (FREIRE, 2014).

\section{Metodologia}

Dando continuidade à pesquisa relatada acima, a presente investigação realizou uma análise quantitativa, seguida de discussão qualitativa, do desenvolvimento musical dessas 25 crianças atendidas em Musicoterapia Improvisacional Musicocentrada. O desenvolvimento musical foi uma nova variável, não observada durante os atendimentos, nem descrita na dissertação de Mestrado de Freire (2014).

\subsection{Instrumentos de Avaliação}

Para as análises quantitativas desta pesquisa, foram utilizados quatro instrumentos de avaliação, que serão apresentados adiante, explicitando-se a forma como foram aplicados:

1. Children Global Assessment Scale (CGAS) [Escala de Avaliação Global de Crianças] (SHAFFER et al., 1983);

2. Clinical Global Impression [Escala de Impressão Clínica Global] (CGI) (GUY, 2000);

3. Autism Treatment Evaluation Checklist (ATEC) [Escala de Avaliação de Tratamento de Autismo] (ARI, 2007); 
Musicoterapia Improvisacional Musicocentrada e Crianças com Autismo: Relações... Marina Horta Freire • Maria Betânia Parizzi • Jéssica Martelli • Renato Tocantins Sampaio

4. Escala de Desenvolvimento Musical de Crianças com Autismo (DEMUCA), (OLIVEIRA, 2015; FREIRE et al., 2019).

As três primeiras escalas - CGAS, CGI e ATEC - foram trazidas para esta pesquisa em dados pré-coletados na dissertação de Mestrado realizada por Freire (2014), descrita no item 3 deste texto. Esses dados haviam sido coletados nas entrevistas da musicoterapeuta com os pais das crianças, antes e após os atendimentos musicoterapêuticos.

A escala CGAS é um instrumento criado para mensurar o funcionamento global da criança, independente de diagnóstico, tendo sido adaptada da Escala de Avaliação Global (Global Assessment Scale - GAS) para adultos (SHAFFER et al., 1983). Possui pontuação de 0 a 100, em níveis descritivos que aumentam de 10 em 10 pontos, e é diretamente proporcional ao grau de desempenho da criança, considerando-se quatro áreas: em casa, na escola, no lazer e com os pares.

A escala CGI é uma ferramenta de avaliação que mensura a impressão global do avaliador em relação ao paciente e seu estado mental no momento da consulta (GUY, 2000). Apresenta duas subescalas de sete pontos cada: (a) CGI-Gravidade, que vai de 1 (ausência de sintomas) até 7 (extremamente doente) e (b) CGIMelhora clínica, que vai de 1 (muito melhor) até 7 (muito pior). A segunda escala só é aplicada a partir da segunda avaliação do paciente.

A escala ATEC é uma ferramenta de avaliação da efetividade de tratamentos para autismo, criada para ser mais sensível às melhoras na condição clínica da criança do que as escalas diagnósticas. É uma escala diretamente proporcional aos sintomas e comprometimentos do indivíduo, dividida em quatro subescalas que abrangem as áreas afetadas pelo autismo: Fala/Linguagem/ Comunicação, Sociabilidade, Percepção Sensorial/Cognição e Saúde/Aspectos físicos/Comportamentos (ARI, 2007).

A Escala DEMUCA foi a ferramenta escolhida para trazer uma nova variável de pesquisa. Ela é um instrumento brasileiro, criado 
Musicoterapia Improvisacional Musicocentrada e Crianças com Autismo: Relações... Marina Horta Freire • Maria Betânia Parizzi • Jéssica Martelli • Renato Tocantins Sampaio

especificamente para avaliar o desenvolvimento musical de crianças com autismo, com evidências de validação apresentadas pelos autores do presente estudo em trabalho anterior (FREIRE et al., 2019). A escala é composta por seis categorias: Comportamentos Restritivos, Interação Social/Cognição, Percepção/Exploração Rítmica, Percepção/Exploração Sonora, Exploração Vocal e Movimentação Corporal com a Música. Cada item apresenta três níveis de respostas: "não", "pouco" e "muito", que são mensurados de forma diretamente proporcional ao desempenho da criança (FREIRE et al., 2019). Além dos valores de cada categoria, a escala retorna dois totais: (1) Total Categorias Musicais (soma de Percepção/Exploração Rítmica até Movimentação Corporal com a Música) e (2) Total da Escala (soma de todas as categorias). Para esta investigação, a aplicação da escala foi feita por duas graduandas bolsistas de iniciação científica (uma graduanda em Musicoterapia e uma graduanda em Licenciatura em Música), por meio da avaliação cega de vídeos-excertos iniciais e finais, como será explicado a seguir.

\subsection{Vídeos-excertos}

A aplicação da Escala DEMUCA, para análise do desenvolvimento musical das crianças atendidas, foi realizada utilizando-se trechos de vídeos pré-filmados de sessões de Musicoterapia dessas crianças. Para isso, foram confeccionados vídeos-excertos de cenas das primeiras e últimas sessões, com a duração entre 2 minutos a 2 minutos e 30 segundos para cada vídeo. Esse processo de confecção se deu em três etapas:

1. Foram selecionados vídeos iniciais e finais de três participantes aleatórios ${ }^{5}$ para um teste piloto, a fim de avaliar a viabilidade dos recortes dos vídeos para aplicação da Escala DEMUCA. A análise e o recorte das cenas seguiram dois critérios pré-estabelecidos pelos pesquisadores: (a) atividades e comportamentos mais predominantes durante as

$\overline{5 \mathrm{~A} \text { aleatoriedade se }}$ deu por sorteio no programa Microsoft Office Excel. 
sessões e (b) a realização das atividades descritas na escala. Priorizou-se utilizar trechos da primeira e da última sessão, mas, em casos de falhas em áudios e imagens desfocadas, também foram utilizados trechos da segunda e da penúltima sessão da criança.

2. Os vídeos-excertos dos três pacientes pilotos foram assistidos e discutidos pelas pesquisadoras e percebeu-se que eles poderiam ser utilizados para o preenchimento da DEMUCA.

3. Após o teste piloto, foram organizados os vídeos das outras 22 crianças. Assim, no total, formou-se um banco de dados de 25 pacientes em 50 vídeos-excertos (incluídos os três primeiros vídeos testados). Cada vídeo-excerto foi assistido e discutido pelas duas primeiras autoras deste estudo, que anotaram a descrição de todos os comportamentos observados em cada vídeo para futuras avaliações qualitativas.

Depois de prontos, os vídeo-excertos foram encaminhados para as avaliadoras (duas graduandas bolsistas de iniciação científica, conforme explicado anteriormente), para aplicação da Escala DEMUCA. Os vídeos estavam sem identificação e em ordem aleatória, ou seja, as avaliadoras não tinham conhecimento do nome das crianças nem quais vídeos eram de sessões iniciais ou finais.

\subsection{Análises Estatísticas}

As análises estatísticas foram realizadas em duas etapas: (1) análises de comparação do nível de desenvolvimento musical no início e no fim do tratamento e (2) análises de correlação entre o desenvolvimento musical (Escala DEMUCA) e os ganhos terapêuticos encontrados (Escalas CGAS, CGI e ATEC). Para fins estatísticos, a distribuição de todos os conjuntos de dados foi classificada como não normal, por isso os métodos de análise adotados foram os não paramétricos (DANCEY; REIDY, 2006). 
Musicoterapia Improvisacional Musicocentrada e Crianças com Autismo: Relações... Marina Horta Freire • Maria Betânia Parizzi • Jéssica Martelli • Renato Tocantins Sampaio

Para as análises de comparação do desenvolvimento musical no início e no final do tratamento musicoterapêutico, foram utilizados os dados obtidos com a aplicação da Escala DEMUCA para os vídeos-excertos iniciais e finais das 25 crianças avaliadas. Foi aplicado o teste de Wilcoxon às pontuações das escalas nas duas condições (inicial e final). Esse teste permite o cálculo das diferenças entre duas amostras relacionadas, sendo que quanto maior o valor do resultado, maior a diferença entre as variáveis (DANCEY; REIDY, 2006).

Para as análises de correlação, foram utilizados o delta de desenvolvimento musical (diferença entre as pontuações inicial e final da Escala DEMUCA) e o delta de melhora terapêutica (diferença entre as pontuações final e inicial das escalas ATEC, CGAS e CGI). Os deltas foram calculados de forma a sempre retornarem um valor diretamente proporcional ao desenvolvimento e à melhora da criança. A relação entre os deltas foi analisada por meio do cálculo estatístico de correlação de Spearman. A classificação da força das correlações seguiu os parâmetros descritos por Dancey e Reidy $(2006)^{6}$.

Todas análises estatísticas foram feitas utilizando-se o software GraphPad Prism 5.0. Para que o resultado fosse considerado estatisticamente significativo, buscou-se o nível de significância de $p$ menor ou igual a 0,05 para todas as análises.

\section{Resultados e Discussão}

\subsection{Análises Comparativas do Desenvolvimento Musical}

Em linhas gerais, a comparação quantitativa entre desenvolvimento musical das crianças atendidas em Musicoterapia Improvisacional Musicocentrada no início e no fim do tratamento, por meio da Escala DEMUCA, mostrou maior nível de

6 Correlação fraca (coeficiente entre 0,1 e 0,3); correlação moderada (coeficiente entre 0,4 e 0,6); e correlação forte (coeficiente entre 0,7 e 0,9$)$. 
Musicoterapia Improvisacional Musicocentrada e Crianças com Autismo: Relações... Marina Horta Freire • Maria Betânia Parizzi • Jéssica Martelli • Renato Tocantins Sampaio

desenvolvimento musical dessas crianças nas sessões finais do que nas sessões iniciais.

Quadro 1: Comparação entre o nível de desenvolvimento musical inicial e final das crianças estudadas $(n=25)$.

\begin{tabular}{|c|c|c|c|c|c|c|c|}
\hline \multirow[b]{2}{*}{ ESCALA DEMUCA } & \multicolumn{3}{|c|}{ INICIAL } & \multicolumn{3}{|c|}{ FINAL } & \multirow{2}{*}{$\begin{array}{c}\underset{\mathbf{X}}{\text { INICIAL }} \\
\text { FINAL } \\
\text { Valor de } p\end{array}$} \\
\hline & Mediana & Média & (DP) & Mediana & Média & (DP) & \\
\hline Comportamentos Restritivos & 13 & 12,76 & $(1,739)$ & 14 & 13,72 & $(0,542)$ &, $0016 * *$ \\
\hline Interação Social/Cognição & 9 & 7,96 & $(2,263)$ & 10 & 9,04 & $(2,031)$ &, $0042 * *$ \\
\hline $\begin{array}{c}\text { Percepção/Exploração } \\
\text { Rítmica }\end{array}$ & 6 & 7,68 & $(5,596)$ & 12 & 10,28 & $(5,474)$ & ,0292* \\
\hline Percepção/Exploração Sonora & 6 & 5,68 & $(4,289)$ & 7 & 6,640 & $(4,499)$ & 0793 \\
\hline Exploração Vocal & 2 & 2,40 & $(2,198)$ & 3 & 4,28 & $(4,026)$ &, $0265^{*}$ \\
\hline $\begin{array}{c}\text { Movimentação Corporal } \\
\text { com a Música }\end{array}$ & 1 & 1,36 & $(1,319)$ & 2 & 2,92 & $(2,532)$ &, $0024 * *$ \\
\hline Total das Categorias Musicais & 15 & 17,12 & $(9,846)$ & 24 & 24,12 & $(11,15)$ &, $0031 * *$ \\
\hline Total da Escala & 35 & 37,88 & $(11,90)$ & 46 & 46,88 & $(12,90)$ &, $0013 * *$ \\
\hline
\end{tabular}

$D P=$ desvio padrão. Fonte: os autores

* Diferenças significativas com $p \leq 0,05$, destacadas em azul médio;

** Diferenças significativas com $p \leq 0,01$, destacadas em azul escuro.

Conforme pode ser observado no Quadro 1, a mediana e a média das avaliações iniciais foram sempre menores do que a mediana e a média das avaliações finais, para todas as categorias e os valores totais da Escala DEMUCA. Apenas para a categoria Percepção/Exploração Sonora não houve diferença estatística significativa entre as avaliações iniciais e finais. A diferença estatística foi significativa, com $p \leq 0,05$, para duas categorias da escala: Percepção/Exploração Rítmica e Exploração Vocal. Essa diferença apresentou ainda maior significância estatística, com $p \leq 0,01$, para as categorias: Comportamentos Restritivos, Interação Social/ Cognição, Movimentação Corporal com a Música, para o Total das Categorias Musicais e o Total da escala.

Esses resultados sugerem que as crianças se desenvolveram musicalmente com as sessões de Musicoterapia, mostrando que a Musicoterapia Improvisacional Musicocentrada pode apresentar ligações com o desenvolvimento musical das crianças atendidas. Porém, outras análises de correlação também se fizeram 
Musicoterapia Improvisacional Musicocentrada e Crianças com Autismo: Relações...

Marina Horta Freire • Maria Betânia Parizzi • Jéssica Martelli • Renato Tocantins Sampaio

necessárias para se investigar essa hipótese e serão apresentadas no item 3.2.

A única categoria da Escala DEMUCA que não retornou resultados estatisticamente significativos para o desenvolvimento musical das crianças atendidas foi a Percepção/Exploração Sonora. Por meio da descrição dos comportamentos observados nos vídeos-excertos, percebemos que as crianças atendidas aprimoraram vários quesitos do desenvolvimento musical, e entre eles estão incluídos a percepção e a exploração de elementos sonoros, como timbres, planos de altura, contraste de intensidade e senso de conclusão. Porém, essa evolução pode ter sido pequena, e a escala pode não ter sido sensível o suficiente para mensurar esse desenvolvimento. No futuro, outras investigações podem ser realizadas para verificar o desenvolvimento musical das crianças atendidas, especificamente em relação à exploração e à percepção sonora de cada criança.

\subsection{Correlações Entre Desenvolvimento Musical e Ganhos Terapêuticos}

O desenvolvimento musical encontrado foi correlacionado com os ganhos terapêuticos encontrados em dados pré-coletados que avaliavam: a melhora clínica funcional dessas crianças (CGAS), a diminuição da gravidade do seu quadro clínico (CGI-Gravidade), sua melhora clínica geral (CGI-Melhora) e a evolução do tratamento do autismo nas principais áreas afetadas por este transtorno (ATEC). Os resultados são apresentados a seguir, separando as avaliações clínicas gerais da criança (CGAS e CGI) da avaliação específica de autismo (ATEC). 
Musicoterapia Improvisacional Musicocentrada e Crianças com Autismo: Relações... Marina Horta Freire • Maria Betânia Parizzi • Jéssica Martelli • Renato Tocantins Sampaio

Quadro 2: Coeficientes de correlação entre o desenvolvimento musical (Escala DEMUCA) e funcionamento, gravidade e melhora clínica das crianças estudadas (Escalas CGAS e CGI).

\begin{tabular}{|c|c|c|c|c|}
\hline \multicolumn{2}{|c|}{ ESCALA DEMUCA } & CGAS & $\begin{array}{c}\text { CGI } \\
\text { Gravidade }\end{array}$ & $\begin{array}{c}\text { CGI } \\
\text { Melhora } \\
\text { Clínica }\end{array}$ \\
\hline $\begin{array}{l}\text { Comportamentos } \\
\text { restritivos }\end{array}$ & $\begin{array}{c}\text { Coeficiente de correlação } \\
\text { Valor de } p\end{array}$ & $\begin{array}{r}, 687^{* \star} \\
0\end{array}$ & $\begin{array}{r}, 377^{\star} \\
0,032\end{array}$ & $\begin{array}{r}-0,333 \\
0,052\end{array}$ \\
\hline $\begin{array}{l}\text { Interação Social } \\
\text { /Cognição }\end{array}$ & $\begin{array}{c}\text { Coeficiente de correlação } \\
\text { Valor de } p\end{array}$ & $\begin{array}{l}\mathbf{5 9 2}^{\star \star} \\
0,001\end{array}$ & $\begin{array}{r}, 626^{* \star} \\
0\end{array}$ & $\begin{array}{l}\mathbf{4 5 7}^{*} \\
0,011\end{array}$ \\
\hline $\begin{array}{c}\text { Percepção/Exploração } \\
\text { Rítmica }\end{array}$ & $\begin{array}{c}\text { Coeficiente de correlação } \\
\text { Valor de } p\end{array}$ & $\begin{array}{r}677^{\star \star} \\
0\end{array}$ & $\begin{array}{r}\mathbf{6}^{649} 9^{* *} \\
0\end{array}$ & $\begin{array}{r}\mathbf{4 6 5}^{* \star} \\
0,01\end{array}$ \\
\hline $\begin{array}{l}\text { Percepção/Exploração } \\
\text { Sonora }\end{array}$ & $\begin{array}{c}\text { Coeficiente de correlação } \\
\text { Valor de } p\end{array}$ & $\begin{array}{r}, 637^{\star \star} \\
0 \\
\end{array}$ & $\begin{array}{r}, 676^{* *} \\
0\end{array}$ & $\begin{array}{r}, 716^{\star *} \\
0 \\
\end{array}$ \\
\hline Exploração Vocal & $\begin{array}{c}\text { Coeficiente de correlação } \\
\text { Valor de } p\end{array}$ & $\begin{array}{c}\mathbf{3 4 5}^{\star} \\
0,046\end{array}$ & $\begin{array}{r}-0,323 \\
0,058\end{array}$ & $\begin{array}{r}\mathbf{3 5 6}^{*} \\
0,04\end{array}$ \\
\hline $\begin{array}{c}\text { Movimentacão corporal } \\
\text { com Música }\end{array}$ & $\begin{array}{c}\text { Coeficiente de correlação } \\
\text { Valor de } p\end{array}$ & $\begin{array}{r}-0,039 \\
0,427\end{array}$ & $\begin{array}{r}\mathbf{3 8 6}^{*} \\
0,028\end{array}$ & $\begin{array}{r}-0,058 \\
0,391\end{array}$ \\
\hline $\begin{array}{c}\text { Total das Categorias } \\
\text { Musicais }\end{array}$ & $\begin{array}{c}\text { Coeficiente de correlação } \\
\text { Valor de } p\end{array}$ & $\begin{array}{r}, 705^{* *} \\
0\end{array}$ & $\begin{array}{r}, 654^{* *} \\
0\end{array}$ & $\begin{array}{r}, 659^{* *} \\
0\end{array}$ \\
\hline Total da Escala & $\begin{array}{c}\text { Coeficiente de correlação } \\
\text { Valor de } p\end{array}$ & $\begin{array}{r}\mathbf{7 3 1}^{* \star} \\
0\end{array}$ & $\begin{array}{r}\mathbf{6 7 9}^{* *} \\
0\end{array}$ & $\begin{array}{r}, 656^{\star *} \\
0\end{array}$ \\
\hline
\end{tabular}

Em relação às avaliações clínicas gerais da criança (Quadro 2), das 24 relações analisadas entre as categorias da Escala DEMUCA e as escalas CGAS e CGI, foram encontradas 20 relações estatisticamente significativas. Quanto à força dessas relações, para a maioria dos resultados ela foi moderada (13 correlações). Além dessas, houve 4 correlações fracas e 3 fortes. As correlações com mais força foram as estabelecidas entre CGAS e totais da Escala DEMUCA e a estabelecida entre CGI-Melhora e Percepção/Exploração Sonora. Todas as correlações estatisticamente significativas apresentaram coeficientes positivos. Esses resultados indicam que, mesmo em relações moderadas ou fracas, quanto maior foi o desenvolvimento musical geral das crianças estudadas, melhor estava sua funcionalidade geral (em casa, na escola, no lazer e com os pares), e também que quanto maior foi o índice de melhora clínica da criança após as sessões de Musicoterapia, maior também foi o seu desenvolvimento musical. É importante realçar que mesmo que a diferença entre as condições inicial e final da categoria Percepção/ 
Musicoterapia Improvisacional Musicocentrada e Crianças com Autismo: Relações... Marina Horta Freire • Maria Betânia Parizzi • Jéssica Martelli • Renato Tocantins Sampaio

Exploração Sonora não tenha apresentado significância estatística, a proporção com que as crianças melhoraram sua percepção e exploração dos sons se relacionou com o nível de sua melhora clínica geral.

Observando os resultados por escala (ainda no Quadro 2), nota-se que: (1) a melhora clínica funcional das crianças (CGAS) correlacionou-se com todas categorias da Escala DEMUCA, exceto com Movimentação Corporal com Música; (2) a diminuição da gravidade do quadro clínico das crianças (CGI-Gravidade) estabeleceu relação positiva com todas categorias da Escala DEMUCA, exceto Exploração Vocal; (3) e a melhora clínica geral das crianças (CGI-Melhora) também correlacionou-se positivamente com todas categorias da Escala DEMUCA, exceto Comportamentos Restritivos e Movimentação Corporal com Música.

A categoria Movimentação Corporal com Música da Escala DEMUCA foi a que menos apresentou correlações significativas com CGAS e CGI (apenas uma correlação com significância estatística fraca). Esse resultado pode sugerir que o desenvolvimento da capacidade de se movimentar acompanhando a música é a área do desenvolvimento musical que está menos ligada à melhora de funcionalidade e do quadro clínico das crianças estudadas. Uma outra hipótese relaciona-se à abordagem musicoterapêutica utilizada, pautada na improvisação musical livre e não em atividades musicais focadas em movimentos (como as danças e canções de ação comumente utilizadas com crianças). Assim, a abordagem improvisacional não privilegiou nem estimulou esta categoria da Escala DEMUCA, o que pode ter levado à baixa correlação encontrada. 
Musicoterapia Improvisacional Musicocentrada e Crianças com Autismo: Relações... Marina Horta Freire • Maria Betânia Parizzi • Jéssica Martelli • Renato Tocantins Sampaio

\section{Quadro 3: Correlação entre desenvolvimento musical (Escala DEMUCA) e evolução de tratamento do autismo (ATEC) das crianças estudadas}

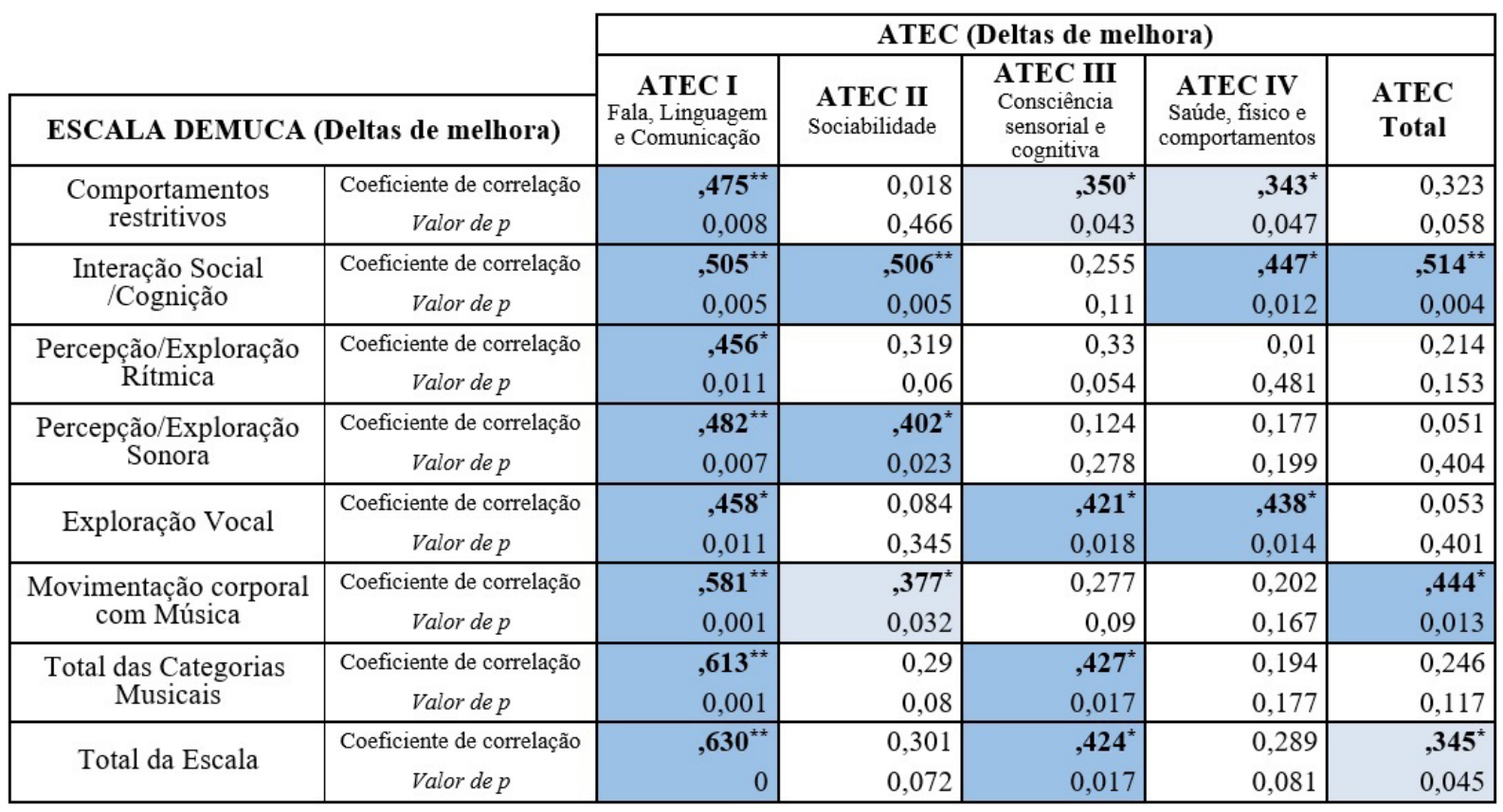

${ }^{*} p \leq 0,05 ;{ }^{* *} p \leq 0,01$. Fonte: os autores

Em relação à avaliação de tratamento do autismo (Quadro 3), das 40 relações analisadas entre as categorias da Escala DEMUCA e as subescalas ATEC, foram encontradas 21 relações estatisticamente significativas. Quanto à força dessas relações, a maioria foi moderada (17) e apenas 4 delas foram fracas. Destaca-se que todas subescalas ATEC estabeleceram pelo menos 2 correlações moderadas com a Escala DEMUCA. Todas as correlações apresentaram coeficiente positivo. Esses resultados mostram que, para as relações significativas, mesmo que as relações não sejam fortes, quanto maior o desenvolvimento musical da criança, maior também pode ter sido a melhora dos seus sintomas autísticos, e vice-versa.

Faz-se importante ressaltar que a subescala que avaliou melhora de fala, linguagem e comunicação (ATEC I) foi a única a apresentar correlações significativas ( $p \leq 0,05$, em força moderada) com todas as categorias da Escala DEMUCA (Quadro 3). Esse achado sugere que o desenvolvimento da musicalidade e da 
Musicoterapia Improvisacional Musicocentrada e Crianças com Autismo: Relações... Marina Horta Freire • Maria Betânia Parizzi • Jéssica Martelli • Renato Tocantins Sampaio

capacidade de comunicação da criança estão relacionados, como apontam diversos estudos sobre o processamento neural infantil (WAN; SCHLAUG, 2010; SARAPA; KATUSIC, 2012; HUTKA et al., 2013; ROCHA; BOGGIO, 2013).

Esses resultados fomentam reflexões para as quais torna-se importante retomar os teóricos da Musicalidade Comunicativa apresentados na introdução deste artigo (MALLOCH; TREVARTHEN, 2009; MALLOCH; TREVARTHEN, 2018). Esses autores já estabeleceram essa relação entre desenvolvimento musical e da comunicação, explicitando suas interligações e o papel fundamental da musicalidade da comunicação mãe-bebê no desenvolvimento da linguagem para toda criança, desde o seu nascimento. É possível que a Musicoterapia Improvisacional Musicocentrada possa espeIhar essa Musicalidade Comunicativa da relação mãe-bebê na relação terapeuta-paciente, incentivando e motivando a criança com autismo a desenvolver a linguagem e a comunicação (TREVARTHEN, 2002).

Assim, resultados quantitativos vão em direção a estudos qualitativos que correlacionam esse tipo de abordagem musicoterapêutica ao desenvolvimento de crianças com autismo, fundamentados na teoria da Musicalidade Comunicativa (ROBARTS, 1998; WIGRAM; ELEFANT, 2009; ANSDELL, 2014). Neste contexto, é interessante ressaltar os musicoterapeutas Nordoff-Robbins, considerados os pioneiros da abordagem musicocentrada de Musicoterapia, que focavam a improvisação musical clínica nos potenciais musicais da criança com autismo, como choro, gritos, risos, vocalizações, pulso, expressões faciais e gestos corporais (características semelhantes às da Musicalidade Comunicativa), para sintonizar com a criança na exata dimensão de seus sons e gestos e, assim, engajá-la na comunicação musical espontânea, proporcionando intercomunicação e reciprocidade (NORDOFF; ROBBINS, 2007).

Os resultados do presente estudo também vão ao encontro de Covre (2015), que se fundamenta em relações da Musicoterapia com a comunicação (dentre elas a Musicalidade Comunicativa) 
Musicoterapia Improvisacional Musicocentrada e Crianças com Autismo: Relações... Marina Horta Freire • Maria Betânia Parizzi • Jéssica Martelli • Renato Tocantins Sampaio

para estudar o processo musicoterapêutico de 3 crianças pré-escolares com distúrbios de linguagem. Dentre as avaliações utilizadas, a autora verificou quantitativamente o desenvolvimento de musicalidade, comunicação receptiva e comunicação expressiva dessas crianças. Os resultados dialogam com a presente discussão, pois demonstraram evolução das crianças atendidas tanto em sua capacidade comunicativa como em sua musicalidade, após as intervenções musicoterapêuticas, revelando as contribuições paralelas da Musicoterapia para o desenvolvimento da musicalidade e da comunicação. De acordo com a autora, todas as principais áreas do desenvolvimento humano associadas à comunicação - habilidades cognitivas/de processamento, características culturais, comunicação receptiva e comunicação expressiva - estão presentes na improvisação musical clínica e podem por ela ser impulsionadas (COVRE, 2015).

Podem ser levantadas algumas hipóteses para esses resultados quantitativos alcançados (correlações estatísticas, em sua maioria, fracas e moderadas - Quadros 2 e 3):

1. O escopo das escalas: as escalas de avaliação clínica e de tratamento não têm relações diretas com as questões musicais avaliadas pela Escala DEMUCA, o que pode contribuir para as relações estabelecidas não serem tão fortes quanto correlações que analisam constructos semelhantes;

2. A diferença entre os contextos e formas de coleta de dados: as avaliações do desenvolvimento musical foram aplicadas em observação cega de vídeos-excertos das próprias sessões musicoterapêuticas, por estudantes da área, enquanto as outras avaliações foram respondidas pelos pais em entrevistas, fora das sessões, o que pode ter favorecido o afastamento entre os dados analisados;

3. Os objetivos musicoterapêuticos traçados: as sessões de Musicoterapia analisadas não haviam sido direcionadas para o desenvolvimento musical das crianças atendidas, tendo-se dado atenção ao fato somente depois do fim dos 
Musicoterapia Improvisacional Musicocentrada e Crianças com Autismo: Relações... Marina Horta Freire • Maria Betânia Parizzi • Jéssica Martelli • Renato Tocantins Sampaio

atendimentos. Os objetivos musicoterapêuticos buscavam alcançar os ganhos terapêuticos, principalmente, melhoras na comunicação e na interação social das crianças atendidas, por meio do incentivo ao desenvolvimento de potenciais dessas crianças. Acreditamos que o foco das sessões pode ter influenciado as diferenças entre o delta de melhora terapêutica e o delta de desenvolvimento musical.

Em razão da pouca força das correlações encontradas (Quadros 2 e 3), não foi possível realizar a comparação entre o desenvolvimento musical e a melhora terapêutica por meio de análises estatísticas fatoriais exploratórias ou análises de regressão (as quais permitem avaliar o valor das mudanças de cada variável, dependendo da mudança da outra). As análises de correlação realizadas permitiram somente avaliar que duas variáveis estão associadas entre si e qual a força dessa associação, sem poder se inferir relações de causalidade (DANCEY; REIDY, 2006). Por isso, indica-se que mais pesquisas sejam feitas sobre o tema, de preferência avaliando o desenvolvimento musical em sessões musicoterapêuticas que tenham foco no desenvolvimento musical da criança desde o início do processo terapêutico. Acreditamos que assim poderão ser encontrados resultados mais significativos, que possam corroborar as relações do desenvolvimento musical em crianças autistas com a Musicoterapia e sua importância para o desenvolvimento integral dessas crianças, já vislumbrado pelo presente estudo, não apenas em relação à comunicação.

\section{Considerações Finais}

O presente artigo avaliou o desenvolvimento musical de 25 crianças com autismo atendidas em Musicoterapia Improvisacional Musicocentrada e correlacionou esse desenvolvimento com ganhos terapêuticos das mesmas crianças. Os resultados mostraram que as crianças apresentaram aumento do nível de desenvolvimento musical após o tratamento musicoterapêutico, com 
Musicoterapia Improvisacional Musicocentrada e Crianças com Autismo: Relações... Marina Horta Freire • Maria Betânia Parizzi • Jéssica Martelli • Renato Tocantins Sampaio

diferenças estatísticas significativas para a maioria das categorias analisadas, e que o desenvolvimento musical e os ganhos terapêuticos estabeleceram correlações positivas, em sua maioria moderadas, destacando-se a relação entre o desenvolvimento musical e a melhora da linguagem/comunicação.

Os resultados não mostram a maior força possível de correlação entre desenvolvimento musical e ganhos terapêuticos e não mostraram correlações estatisticamente significativas para todas as subescalas avaliadas. Ainda assim, o predomínio da intensidade moderada, com significância estatística na maior parte das correlações analisadas, pode ser considerado um resultado satisfatório, por terem sido correlacionadas variáveis tão distintas, avaliadas por diferentes avaliadores em diferentes contextos. Além disso, o estudo pode ser considerado importante pela originalidade do tema investigado, com utilização do método quantitativo, apontando uma direção, o início de um caminho, para as conexões diretas que podem ser discutidas entre Musicoterapia e desenvolvimento musical.

Algumas sugestões são feitas para pesquisas futuras em Musicoterapia, tais como investigar o desenvolvimento musical, especificamente em relação à exploração e à percepção sonora; analisar grupos com um maior número de participantes; comparar crianças atendidas com grupo controle; delinear intervenções musicoterapêuticas que tenham foco no desenvolvimento musical do paciente; e aprofundar nas relações entre a comunicação e o desenvolvimento musical, tema que se destacou dentre os resultados encontrados.

As discussões apresentadas implicam na relevância das relações entre Musicoterapia Improvisacional Musicocentrada, desenvolvimento musical e Musicalidade Comunicativa. A improvisação musicoterapêutica, centrada na música, enfatiza o processo individualizado, criativo e integral de cada indivíduo a partir de sua própria musicalidade (NORDOFF; ROBBINS, 2007; ANSDELL, 2014). Ou seja, a partir do nível desenvolvimento musical que o paciente se encontra, ao mesmo tempo em que fortalece as trocas musicais 
Musicoterapia Improvisacional Musicocentrada e Crianças com Autismo: Relações... Marina Horta Freire • Maria Betânia Parizzi • Jéssica Martelli • Renato Tocantins Sampaio

afetivas entre terapeuta e paciente (ROBARTS, 1998; TREVARTHEN, 2002), expandindo o desenvolvimento musical do paciente.

Dessa forma, compreendemos que as experiências improvisacionais coativas em Musicoterapia podem servir tanto como estímulo externo (contato ativo com a música) (PENDEZA; AZEVEDO, 2018), quanto como estímulo interno (criação de estados motivacionais e emocionais ligados à Musicalidade Comunicativa) (TREVARTHEN, 2002; ANSDELL, 2014), podendo concorrer para promover o desenvolvimento musical e o desenvolvimento do indivíduo como um todo. O desenvolvimento musical poderia inclusive ser traçado como um objetivo terapêutico primário em Musicoterapia. Essas discussões se tornam ainda mais relevantes no autismo, uma vez que crianças com autismo podem se beneficiar da música quando ela thes proporciona engajamento afetivo e trocas interpessoais, oportunizando melhoras significativas na comunicação social (TREVARTHEN, 2002; WIGRAM; ELEFANT, 2009; SAMPAIO, 2015).

Além de buscar o aprimoramento de conhecimentos e discussões na área do autismo, a presente pesquisa visou contribuir para as interfaces entre várias áreas do conhecimento, principalmente entre Educação Musical, Musicoterapia e Saúde, tendo em vista a relevância do desenvolvimento musical para o desenvolvimento integral do indivíduo.

\section{Referências}

ANSDELL, Gary. Musical Companionship. In: ANSDELL, Gary. How music helps in Music Therapy and Everyday life. New York: Routledge, 2014. cap. 11, p. 145-155.

AMERICAN PSYCHIATRIC ASSOCIATION (APA). Diagnostic and statistical manual of mental disorders (DSM-5). Arlington: American Psychiatric Publishing, 2014.

AUTISM RESEARCH INSTITUTE (ARI). Autism Treatment Evaluation Checklist (ATEC). Disponível em: https://www.autism.com/ind_atec. Acesso em: 30 jan. 2019. 
Musicoterapia Improvisacional Musicocentrada e Crianças com Autismo: Relações... Marina Horta Freire • Maria Betânia Parizzi • Jéssica Martelli • Renato Tocantins Sampaio

BARCELLOS, Lia Rejane Mendes. A música como metáfora em musicoterapia. 232 f. Tese (Doutorado em Música). Programa de PósGraduação em Música, Centro de Letras e Artes, Universidade Federal do Estado do Rio de Janeiro, Rio de Janeiro, 2009.

BLACKING, John. The value of music in human experience: The 1969 Yearbook of the International Folk Music Council, v. 1, 1969, p. 33-71. [Republished in: BOHLMAN, P.; NETTL, B. (eds.). Music, Culture and Experience: Selected Papers of John Blacking. Chicago: University of Chicago Press, 1995.

COVRE, Josiane Fernanda. Contribuiçõos da Musicoterapia para a comunicação de crianças com alterações da linguagem. 152 f. Dissertação (Mestrado em Música). Programa de Pós-Graduação em Música, Escola de Música e Artes Cênicas, Universidade Federal de Goiás, Goiânia, 2015.

CUERVO, Luciane; ROSAT, Renata Menezes. Abordagem interdisciplinar entre Música e Neurociências: estratégias de fomento e inserção curricular no ensino superior. Orfeu, v. 3, n. 1, p. 172-196, 2018.

DANCEY, Christine; REIDY, John. Estatística sem matemática para psicologia. 3. ed. Porto Alegre: Artmed, 2006.

FIGUEIREDO, Camila Fernandes. A aprendizagem musical de estudantes com autismo por meio da improvisação. $136 \mathrm{f}$. Dissertação (Mestrado em Música). Programa de Pós-Graduação em Música, Universidade Federal do Paraná, Curitiba, 2016.

FINNIGAN, E.; STARR, E. Increasing social responsiveness in a child with autism: a comparison of music and non-music interventions. Autism, v. 14, n. 4, p. 321-348, 2010. DOI 10.1177/1362361309357747.

FREIRE, M. H. Efeitos da Musicoterapia Improvisacional no Tratamento de Crianças com Transtorno do Espectro do Autismo. 74 f. Dissertação (Mestrado em Neurociências). Programa de Pósgraduação em Neurociências, Universidade Federal de Minas Gerais, UFMG, Belo Horizonte, 2014.

FREIRE, Marina; MOREIRA, Aline; KUMMER, Arthur. Protocolo de atendimento de Musicoterapia Improvisacional musicocentrada para 
Musicoterapia Improvisacional Musicocentrada e Crianças com Autismo: Relações... Marina Horta Freire • Maria Betânia Parizzi • Jéssica Martelli • Renato Tocantins Sampaio

crianças com autismo. Revista Brasileira de Musicoterapia, v. XVII, n. 18, p. 104-117, 2015.

FREIRE, Marina; MARTELLI, Jéssica; ESTANISLAU, Gabriel; PARIZZI Betânia. $O$ desenvolvimento musical de crianças com Transtorno do Espectro do Autismo em Musicoterapia: revisão de literatura e relato de caso. Orfeu, v. 3, n. 1, p. 145-171, 2018. Doi: http://dx.doi.or g/10.5965/2525530403012018145.

FREIRE, Marina; MARTELLI, Jéssica; SAMPAIO, Renato; PARIZZI Betânia. Validação da Escala de Desenvolvimento Musical de Crianças com Autismo (DEMUCA): análise semântica, interexaminadores, consistência interna e confiabilidade externa. Opus, v. 25, n. 3, p. 158-187, 2019. Doi: http://dx.doi.org/10.20504/opus2019c2508.

GATTINO, Gustavo. Musicoterapia aplicada à avaliação da comunicação não verbal de crianças com transtornos do espectro autista: revisão sistemática e estudo de validação. 180 f. Tese (Doutorado em Saúde da Criança e do Adolescente). Faculdade de Medicina, Universidade Federal do Rio Grande do Sul, Porto Alegre, 2012.

GERETSEGGER, M.; ELEFANT, C.; MÖSSLER, K. A.; GOLD, C. Music therapy for people with autism spectrum disorder. Cochrane Database of Systematic Reviews, n. 6, p. 1-64, CD004381, 2014. Doi: 10.1002/14651858.CD004381.pub3.

GUY, W. Clinical Global Impressions (CGI) Scale. Modificado de: RUSH, J., et al., Psychiatric Measures. Washington: APA, 2000.

HARGREAVES, David J. The development of artistic and musical competence. In: DELIEGE, I.; SLOBODA, J. A. (Orgs). Musical Beginnings: the origins and development of musical competence. Oxford: Oxford University Press, 1996. p. 145-170.

HUTKA, S., BIDELMAN, G. M., MORENO, S. Brain signal variability as a window into the bidirectionality between music and language processing: moving from a linear to a nonlinear model. Frontiers in Psychology, v. 4, 2013, p. 1-11. Doi: 10.3389/fpsyg.2013.00984 
Musicoterapia Improvisacional Musicocentrada e Crianças com Autismo: Relações... Marina Horta Freire • Maria Betânia Parizzi • Jéssica Martelli • Renato Tocantins Sampaio

KIM, J.; WIGRAN, T.; GOLD, C. The Effects of Improvisational Music Therapy on Joint Attention Behaviors in Autistic Children: A Randomized Controlled Study. J Autism Dev Disord, v. 38, p. 1758-1766, 2008. DOI 10.1007/s10803-008-0566-6.

KIM, J.; WIGRAN, T.; GOLD, C. Emotional, motivational and interpersonal responsiveness of children with autism in improvisational music therapy. Autism SAGE Publications and The National Autistic Society, v. 13, n. 4. p. 389-409, 2009.

LOURO, Viviane dos Santos. Fundamentos da aprendizagem musical da pessoa com deficiência. São Paulo: Editora Som, 2012.

MALLOCH, Stephen. Mothers and Infants and Communicative Musicality. Musicae Scientiae, v. 3, n. 1, p. 29-57, 1999/2000.

MALLOCH, Stephen; TREVARTHEN, Colwyn. (Eds.). Communicative Musicality: exploring the basis of human companionship. Oxford: Oxford University Press, 2009.

MALLOCH, Stephen; TREVARTHEN, Colwyn. The human nature of music. Frontiers in psychology, v. 9, p. 1-21, 2018.

MOLNAR-SZAKACS, I.; HEATON, P. Music: a unique window into the world of autism. Annals of New York Academic Science, v. 1252, p. 318-324, 2012. Doi: 10.1111/j.1749-6632.2012.06465.x.

MOLNAR-SZAKACS, I., WANG, M. J., LAUGESON, E. A., OVER, K., WU, W. L.; PIGGOT, J. Autism, Emotion Recognition and the Mirror Neuron System: The Case of Music. Mcgill Journal of Medicine, v. 12, n. 2, p. 87-98, 2009.

MORENO, Sylvain; BIALYSTOK, Ellen; BARAC, Raluca; SCHELLENBERG, E. Glenn; CEPEDA, Nicholas J. ; CHAU, Tom. Short-Term Music Training Enhances Verbal Intelligence and Executive Function. Psychological Science, v. 22, n. 11, p. 1425-33, 2011. Doi: 10.1177/0956797611416999. MÖSSLER, K., GOLD, C., AßMUS, J. et al. The Therapeutic Relationship as Predictor of Change in Music Therapy with Young Children with Autism Spectrum Disorder. J Autism Dev Disord, v. 49, p. 2795-2809, 2019. DOI 10.1007/s10803-017-3306-y. 
Musicoterapia Improvisacional Musicocentrada e Crianças com Autismo: Relações... Marina Horta Freire • Maria Betânia Parizzi • Jéssica Martelli • Renato Tocantins Sampaio

NORDOFF, P.; ROBBINS, C. Creative Music Therapy: a guide to fostering clinical musicianship. 2. ed. Gilsum: Barcelona Publishers, 2007.

NORTH, Adrian; HARGREAVES, David. The Social and Applied

Psychology of Music. Oxford: University Press, 2008.

OLIVEIRA, Gleisson C. Desenvolvimento musical de crianças autistas em diferentes contextos de aprendizagem: um estudo exploratório. 135 f. Dissertação (Mestrado em Música). Escola de Música, Universidade Federal de Minas Gerais, Belo Horizonte, 2015.

OUIMET, T., FOSTER, N. E. V., TRYFON, A.; HYDE, K. L. Auditory-musical processing in autism spectrum disorders: a review of behavioral and brain imaging studies. Annals of New York Academic Science, v. 1252, p. 325-331, 2012.

PALAZZI, Ambra. Contribuições da Musicoterapia para a díade mãebebê pré-termo na UTI neonatal. 136 f. Dissertação (Mestrado em Psicologia). Instituto de Psicologia, Universidade Federal do Rio Grande do Sul, Porto Alegre, 2016.

PENDEZA, Daniele; AZEVEDO, Graciane Torres. Interfaces entre teorias do desenvolvimento musical e Musicoterapia. Revista Brasileira de Musicoterapia, ano XX, n. 24, p. 91-113, 2018. Disponível em: http:// www.revistademusicoterapia.mus.br/wp-content/uploads/2019/07/5Interfaces-entre-teorias-do-desenvolvimento-musical-e-musicoterapia. pdf. Acesso em: 08 fev. 2019.

ROBARTS, Jacqueline. Music Therapy for Children with Autism. In: TREVARTHEN, C.; AITKEN, K.; PAPOUDI, D., ROBARTS, J. Children with Autism: Diagnosis and Interventions to meet their needs. 2. ed. London and Philadelphia: Jessica Kingsley Publishers, 1998. cap. 11, p. 172-202.

ROCHA, V. C.; BOGGIO, P. S. A música por uma óptica neurocientífica. Per Musi, Belo Horizonte, n. 27, p. 132-140, 2013.

ROJAS, D. G.; ANGULO, G. P.; RODRÍGUES, R. M. S. Efectos de la Musicoterapia em el Trastorno de Espectro Autista. Revista de Educación Inclusiva, v. 11, n. 1, p. 175-192, 2018. 
Musicoterapia Improvisacional Musicocentrada e Crianças com Autismo: Relações... Marina Horta Freire • Maria Betânia Parizzi • Jéssica Martelli • Renato Tocantins Sampaio

SAMPAIO, Renato Tocantins. Avaliação da Sincronia Rítmica em Crianças com Transtorno do Espectro do Autismo em Atendimento Musicoterapêutico. 157 f. Tese (Doutorado em Neurociências). Instituto de Ciências Biológicas, Universidade Federal de Minas Gerais, Belo Horizonte, 2015.

SANTOS, Claudia Eboli C. Potencialidades e talento: um estudo sobre as habilidades musicais em crianças com transtornos do espectro autista. In: SIMPÓSIO BRASILEIRO DE PÓS-GRADUANDOS EM MÚSICA, 3., Rio de Janeiro. Anais... Rio de Janeiro: Unirio, 2014. p. 267-275.

SANTOS, Claudia Eboli C. A música como ferramenta de desenvolvimento para crianças autistas: um estudo na interface da musicoterapia com a educação musical à luz dos conceitos de Vigotski. 246 f. Tese (Doutorado em Música). Centro de Letras e Artes, Universidade Federal do Estado do Rio de Janeiro (UNIRIO), Rio de Janeiro, 2018.

SARAPA, K. B.; KATUSIC, A. H. Application of music therapy in children with autistic spectrum disorder. Croatian review of rehabilitation research, v. 48, n. 2, p. 124-129, 2012.

SHAFFER, D.; GOULD, M. S.; BRASIC, J.; AMBROSINI, P.; FISHER, P.; BIRD, H.; ALUWAHLIA, S. A. Children's Global Assessment Scale (CGAS). Archives of General Psychiatry, v. 40, p. 1228-1231, 1983.

SIMPSON, K.; KEEN, D. Music interventions for children with Autism: narrative review of the literature. J Autism Dev Disord, v. 41, n. 11, p. 1507-14, 2011. DOI 10.1007/s10803 -010-1172-y.

THAUT, Michael H. Measuring Musical Responsiveness in Autistic Children: a comparative analysis of improvised musical tone sequences of autistic, normal, and mentally retarded individuals. J Autism Dev Disord, v. 18, n. 4, p. 561-571, 1988. DOI 10.1007/BF02211874.

THAUT, Michael H (Ed). Part 10 Music Therapy. In: HALLAM, Susan; CROSS, Ian; THAUT, Michael. The Oxford Handbook of Music Psychology. 2. ed. New York: Oxford University Press, 2016. Part 10, p. 804-875. 
Musicoterapia Improvisacional Musicocentrada e Crianças com Autismo: Relações... Marina Horta Freire • Maria Betânia Parizzi • Jéssica Martelli • Renato Tocantins Sampaio

TREVARTHEN, Colwyn. Autism, Sympathy of Motives and Music Therapy.

Presses Universitaires de France, v. 54, p. 86-99, 2002.

WAN, C.; SCHLAUG, G. Neural pathways for language in autism: the potential for music based treatments. Future Neurol, v. 5, n. 6, p. 797-805, 2010.

WIGRAM, Tony; ELEFANT, Cochavit. Therapeutic Dialogues in Music: Nurturing Musicality of Communication in Children with Autistic Spectrum Disorder and Rett Syndrome. In: MALLOCH, S.; TREVARTHEN, C. (Eds.). Communicative Musicality: exploring the basis of human companionship. Oxford: Oxford University Press, 2009. cap. 19, p. 423446.

ZORZAL, Ricieri Carlini. The Psychologyof Music, editado por Diana Deutsch, $3^{a}$ edição: resenha dos capítulos 10 a 13. Revista Opus, v. 22, n. 1, p. 373-386, jun. 2016.

\section{Aprovação do Comitê de Ética em Pesquisa}

Esta pesquisa foi autorizada pelo Comitê de Ética em Pesquisa da UFMG, sob o número CAAE 86460518.5.0000.5149.

\section{Publisher}

Universidade Federal de Goiás. Escola de Música e Artes Cênicas. Programa de Pós-graduação em Música. Publicação no Portal de Periódicos UFG.

As ideias expressadas neste artigo são de responsabilidade de seus autores, não representando, necessariamente, a opinião dos editores ou da universidade. 